\title{
A REVIEW ON MULTIQUERY SYSTEM FOR CONTENT BASED IMAGE RETRIEVAL
}

\author{
Meenu ${ }^{(1)}$, Sonika Jindal ${ }^{(2)}$ \\ ${ }^{(1)}$ Research Scholar, Department of Computer Science Engineering, SBSSTC, Ferozepur \\ kalrameenu1991@gmail.com \\ ${ }^{(2)}$ Assistant Professor, Department of Computer Science Engineering, SBSSTC, Ferozepur \\ sonikamanoj@gmail.com
}

\begin{abstract}
In recent years, very large collections of images and videos have grown rapidly. In parallel with this growth, content-based retrieval and querying the indexed collections are required to access visual information. Two of the main components of the visual information are texture and color. In this thesis, a content-based image retrieval system is presented that computes texture and color similarity among images. Content based image retrieval from large resources has become an area of wide interest now a days in many applications. To speed up retrieval and similarity computation, the database images are analysed and the extracted regions are clustered according to their feature vectors. This process is performed offline before query processing, therefore to answer a query our system does not need to search the entire database images; instead just a number of candidate images are required to be searched for image similarity.
\end{abstract}

\section{Keywords}

Content based image retrieval (CBIR), color histogram, color, shape, texture features.

\section{INTRODUCTION}

With the recent outburst of multimedia-enabled systems, the need for multimedia retrieval has increased by leaps and

bounds. Due to the complexity of multimedia contents, image understanding is a difficult-albeit-interesting topic of research, within the domain of multimedia retrieval. Extracting valuable knowledge from a large-scale multimedia repository, usually referred to as "multimedia mining", has recently caught up as a domain of interest amongst researchers.Typically, in the development of an image requisition system, semantic image retrieval relies heavily on the related captions, e.g., filenames, categories, annotated key-words, and other manual descriptions.Searching of images is predominantly based upon associated metadata such as keywords, text, etc.The term CBIR describes the process of retrieving desired images from the large collection of database on the basis of features that can be automatically extracted from the images. The ultimate goal of a CBIR system is to avoid the use of textual descriptions in the hunt for an image by the user. Unfortunately, this kind of a textual-based image retrieval system always suffers from two problems: high-priced manual annotation and inaccurate and inconsistent automated annotation. On one hand, the cost associated with manual annotation is prohibitive with regards to a large-scale data set. On the other hand, inappropriate automated annotation yields distorted results for semantic image retrieval. As a result, a number of powerful image retrieval algorithms have been proposed to deal with such problems over the past few years. CBIR is the mainstay of current image retrieval systems. In CBIR, retrieval of image is based on similarities in their contents, i.e., textures, colors, shapes etc., which are considered the lower level features of an image. These conventional approaches for image retrieval are based on the computation of the similarity between the users query and images. In CBIR each image stored in the database, has its features extracted and compared to the features of the query image. Thus, broadly, it involves two processes, viz, feature

extraction and feature matching [8]. Feature extraction involves the image features to a distinguishable extent. Average RGB, Color Moments, Cooccurence, Local Color Histogram, Global Color Histogram and Geometric Momentsare used to extract features from the test image. Feature matching, on the other hand, involves matching the extracted features to yield results that exhibitvisual similarities.

With the advancement in internet and multimedia technologies, a huge amount of multimedia data in the form of audio, video and images has been used in many fields like medical treatment, satellite data, video and still images repositories, digital forensics and surveillance system. This has created an ongoing demand of systems that can store and retrieve multimedia data in an effective way. Many multimedia information storage and retrieval systems have been developed

till now for catering these demands. The most common retrieval systems are Text Based Image Retrieval (TBIR) systems, where the search is based on automatic or manual annotation of images. A conventional TBIR searches the database for the similar text surrounding the image as given in the query string. The commonly used TBIR system is Google Images. The text based systems are fast as the string matching is computationally less time consuming process. However, it is sometimes difficult to express the whole visual content of images in words and TBIR may end up in producing irrelevant results. In addition annotation of images is not always correct and consumes a lot of time. For finding the alternative way of searching and overcoming the limitations imposed by TBIR systems more intuitive and user friendly content based image retrieval systems (CBIR) were developed. A CBIR system uses visual contents of the images described in the form of low level features like color, texture, shape and spatial locations to represent the images in the databases. The system retrieves similar images when an example image or sketch is presented as input to the system. Querying in this way eliminates the need of describing the visual content of images in words and is close to human perception of visual data.

Some of the representative CBIR systems are Query by Image Content (QBIC). 


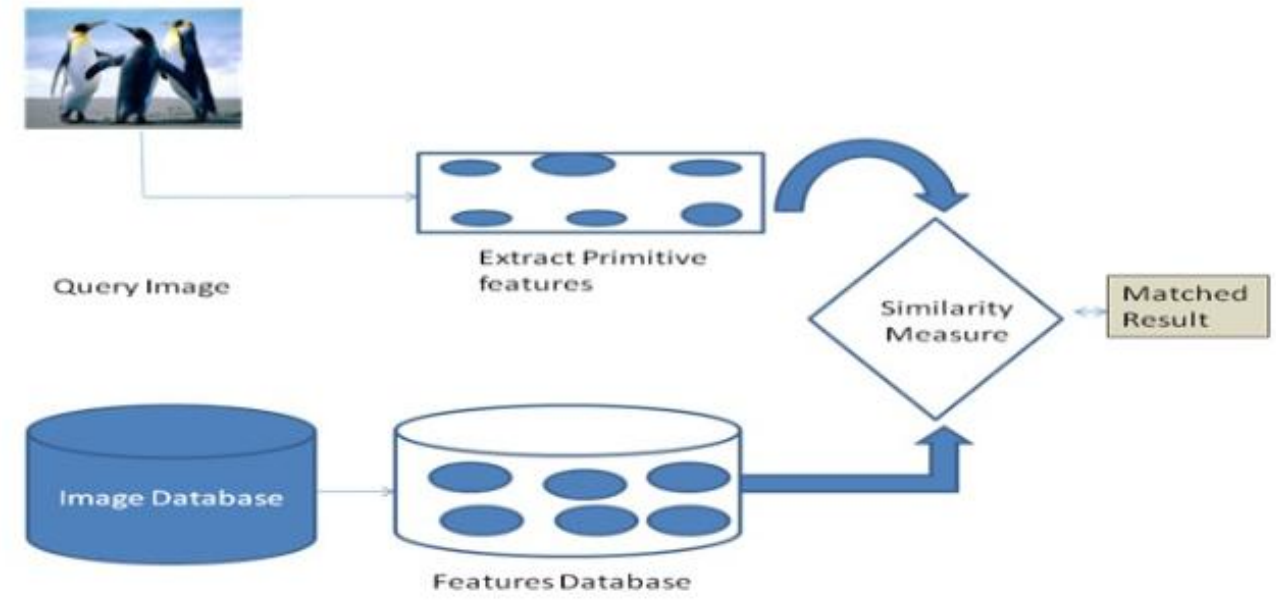

Figure 1. Architecture of CBIR

In a typical CBIR system (Figure 1), image low level features like color, texture, shape and spatial locations are represented in the form of a multidimensional feature vector. The feature vectors of images in the database form a feature database. The retrieval process is initiated when a user query the system using an example image or sketch of the object. The query image is converted into the internal representation of feature vector using the same feature extraction routine that was used for building the feature database. The similarity measure is employed to calculate the distance between the feature vectors of query image and those of the target images in the feature database. Finally, the retrieval is performed using an indexing scheme which facilitates the efficient searching of the image database. Recently, user's relevance feedback is also incorporated to further improve the retrieval process in order to produce perceptually and semantically more meaningful retrieval results.

\section{FIELDS OF APPLICATION}

Image retrieval based on content is extremely useful in a plethora of applications such as publishing and advertising, historical research, fashion and graphic design, architectural and engineering design, crime prevention, medical diagnosis, geographical information and remote sensing systems, etc. [5]. A typical image retrieval application example is a design engineer who needs to search his organization database for design projects similar to that required by his clients, or the police seeking to confirm the face of a suspected criminal among faces in the database of renowned criminals. In the commerce department, before trademark is finally approved for use, there is need to find out if such or similar ones ever existed. In hospitals, some ailments require the medical practitioner to search and review similar X-rays or scanned images of a patient before proffering a solution. The most important application, however, is the Web, as big fraction of it is devoted to images, and searching for a specific image is indeed a daunting task. Numerous commercial and experimental CBIR systems are now available, and many web search engines are now equipped with CBIR facilities, as for example Alta Vista, Yahoo and Google [6].

\section{VISUAL CONTENT DESCRIPTOR}

Natural images depicting a complex scene may contain a variety of visual artifacts. CBIR systems represent the visual contents of images in the form of a feature descriptor. A good descriptor should not only be invariant to rotation, scaling and illumination variations but also has high discriminating capability. However, there is a tradeoff between invariance and discriminating power of visual features. Employing features having wide variety of invariance may result in losing the capability to discriminate between most essential properties. Study of invariance is largely investigated in the field of computer vision but is relatively new in image retrieval. A feature descriptor may be local or global. Local descriptors are extracted using a part or region of an image while a global feature uses the visual content of the whole image. A CBIR system which uses region features to represent images is known as Region Based Image Retrieval systems (RBIR). On the other hand CBIR systems utilizing global features for describing images are classified as Global CBIR systems. Local and Global features of an image largely represent color, texture, shape and spatial relationships of different objects in the image. Some widely used color, texture, shape and spatial relationship features are discussed in the following subsections.

\section{COLOR FEATURES}

Color is the most commonly used feature of an image. The perceived color at any pixel of an image is obtained by mixing three preliminary colors in appropriate proportion. The three dimensional color provides more discriminating information than the single dimensional gray level values. Before extracting color descriptor a proper color space must be determined first. Commonly used color spaces for image retrieval application are RGB, CIE L*a*b*, CIE L* $\mathrm{U}^{*} \mathrm{v}^{*}$, HSV and opponent color space. There is no agreement over which color space is best but one of the desirable characteristic of color space for image retrieval task is its uniformity. Uniformity means that the physical distance between any two color pair in the 
color space must be equal to the perceived distance between them. Some commonly used color descriptors are color moments, color histogram, color coherence vector and color correlogram.

\section{TEXTURE FEATURES}

There is no specific definition of texture however one can define texture as the visual pattern that has properties of homogeneity not resulting from the presence of only a single color or intensity. Various techniques for texture analysis have been investigated in the field of computer vision and pattern recognition. The texture extraction techniques can be classified into two categories: statistical and structural. Statistical approaches use intensity distribution of image to extract statistical parameters representing texture of image. Commonly used statistical methods include Fourier power spectra, Co-occurrence matrices, Shift-invariant principal component analysis (SPCA), Tamura feature, Wold decomposition, Markov random field, Fractal model, and Multi-resolution filtering techniques such as Gabor and wavelet transform. Structural methods, including morphological operator and adjacency graph, describe texture by identifying structural primitives and their placement rules. They tend to be most effective when applied to textures that are very regular.

\section{SHAPE FEATURES}

Shape feature provides the most important semantic information about an image. Shape features are usually described using part or region of an image. The accuracy of shape features largely depends upon the segmentation scheme used to divide an image into meaningful objects. However, fast and robust segmentation is difficult to achieve. This limits the shape features only to those retrieval applications where objects or region of images are readily available. The shape descriptors are categorized into two classes: boundary based descriptor and region based descriptor. Some boundary based representative shape description techniques are chain codes, polygonal approximations, Fourier descriptor and finite element model. On the other hand state of the art region based descriptors are statistical moment and area. A good shape feature should be invariant to translation, rotation and scaling. A detail review of shape matching techniques used in image retrieval application can be found in[Mingqiang et al.(2008)].

\section{SPATIAL INFORMATION}

The performance of a image retrieval system can be improved by considering spatial locations of different objects in the image. The spatial location of objects and their relationship can provide useful discriminating information in image retrieval applications. For instance, parts of blue sky and ocean may have similar color histograms, but their spatial locations in images are different. The spatial location matching can be implemented by matching the images based on fixed location similarity. In this approach a similar object lying in different regions of an image cannot be detected. For instance; image having tiger in the left part may not get similarity with images having tiger in the right part of images. To overcome this problem systems compare all region of image with the query object or region. This may result in the increase of response time of the system. The most commonly used techniques for finding spatial location similarity includes $2 \mathrm{D}$ strings, spatial quad-tree and symbolic images.

\section{SIMILARITY MEASURE}

The degree of similarity between query and target images is calculated based on the value of similarity measure. The images are ranked according to their similarity value and presented as output of CBIR system. Often, the choice of similarity measure affects the performance of retrieval system. Many similarity measures have been developed over the years based on the quantitative estimates of the distribution of features in the image. Some of the most commonly used similarity measures employed in CBIR are Euclidean distance, Minkowski- form distance, Histogram intersection distance, Quadratic- form distance, Mahalanobis distance and Kullback Leibler (KL) divergence distance.

\section{PERFORMANCE EVALUATION}

The performance of a retrieval system is evaluated based on several criteria. Some of the commonly used performance measures are average precision, average recall, average retrieval rate and Average Normalized modified Retrieval Rate (ANMRR). All these parameter are computed using precision and recall values computed for each query image. The precision of the retrieval is defined as the fraction of the retrieved images that are indeed relevant for the query:

$$
\text { Precision }=\frac{\text { No. of relevant images retrieved }}{\text { Total no. of images retrieved from the database }}
$$

The recall is the fraction of relevant images that is returned by the query:

$$
\text { Recall }=\frac{\text { No. of relevant images retrieved }}{\text { Total no. of relevant images in the database }}
$$

A good retrieval system should have high values for precision and recall. 


\section{MOTIVATION OF THE WORK}

In the last two decades, CBIR systems have been improved a lot. However, there still remain some problems which have not been answered satisfactorily. First and foremost problem is of semantic gap, which exist between low level feature representation of images and the actual visual perception of the image. Researchers all over the globe are working in the direction of narrowing down this semantic gap. Semantic gap is a big problem which can be seen as a collection of many small problems. In this work, we have identified such problems and tried to provide an effective solution to these problems.

Many of the existing systems attempt to compare the query image with every target image in the database to find the top matching images, resulting in an essentially linear search, which is prohibitive when the database is large. We believe that it is not necessary to conduct a whole database comparison. In fact, it is possible to exploit a priori information regarding the "organization" of the images in the database in the feature space before a query is posed, such that when a query is received, only a part of the database needs to be searched, while a large portion of the database may be eliminated in the search. This certainly saves significant query processing time without compromising the retrieval precision. To speed up the retrieval process, the database images are segmented into distinct regions. A clustering algorithm, definitely the self organizing map (SOM), is used to cluster the image regions into groups. Regions with similar features are grouped together in the same class. This clustering process is performed offline, and each region's indexing data along with its associated class ID is recorded in the index files. To answer a query, the query image is segmented into its regions. The distances between each query region and all class centroids in the database is computed to determine which class of these query regions belong. The similar regions in the database are returned and all the images that have any of these regions are assigned as candidates. The query image is compared to the candidate image set instead of being compared to the whole database image.

\section{CONCLUSIONS}

Content based image retrieval is a challenging method of capturing relevant images from a large storage space. Although this area has been explored for decades, no technique has achieved the accuracy of human visual perception in distinguishing images. Whatever the size and content of the image database is, a human being can easily recognize images of same category. From the very beginning of CBIR research, similarity computation between images used either region based or global based features. Global features extracted from an image are useful in presenting textured images that have no certain specific region of interest with respect to the user. Region based features are more effective to describe images that have distinct regions. Retrieval systems based on region features are computationally expensive because of the need of segmentation process in the beginning of a querying process and the need to consider every image region in similarity computation. In this paper, we presented an architecture of content based image retrieval with its various applications.

\section{REFERENCES}

[1] M. Fakheri, T. Sedghi, Mahrokh G. Shayesteh and Mehdi Chehel Amirani, "Framework for image retrieval using machine learning and statistical similarity matching techniques," IET Image Process., pp. 1-11, 2013.

[2] P. MANIPOONCHELVI and K. MUNEESWARAN, "Multi region based image retrieval system," Indian Academy of Sciences, pp. 333-344, 2014.

[3] H. Jegou, M. Douze, C. Schmid and P. Perez, "Aggregating local descriptors into a compact image representation," IEEE, pp. 3304-3311, 2010.

[4] Yixin Chen, James Z. Wang, and Robert Krovetz, "CLUE: Cluster-Based Retrieval of Images by Unsupervised Learning," IEEE, pp. 1181-101, 2005.

[5] R. Fergus, L. Fei-Fei, P. Perona and A. Zisserman, "Learning Object Categories from Google's Image Search," IEEE, 2005.

[6] Y. Chen, X. Li, A. Dick and Anton van den Hengel, "Boosting Object Retrieval With Group Queries," IEEE, pp. 765768, 2012.

[7] R. Arandjelovi'c and A. Zisserman, "Three things everyone should know to improve object retrieval," IEEE, pp. 29112918, 2012.

[8] M. Perdoch, Ondrej Chum and Jiri Matas, IEEE, pp. 9-16, 2009.

[9] Savvas A. Chatzichristofis and Yiannis S. Boutalis , "CEDD: Color and Edge Directivity Descriptor: A Compact Descriptor for Image Indexing and Retrieval," Springer-Verlag Berlin Heidelberg, pp. 312-322, 2008.

[10] Yixin Chen and James Z. Wang, "A Region-Based Duzzy Feature Matching Approach to content-Based Image Retrival," IEEE, pp. 1252-1267, 2002.

[11] Chuen-Horng Lin, Rong-Tai Chen and Yung-Kuan Chan, "A smart content-based image retrieval system based on color and texture feature," Elsevier, 2008. 
[12] James Z. Wang, , Jia Li and Gio Wiederhold, "SIMPLlcity: Semantics-Sensitive Integrated Matching for Picture Llbraries," IEEE, pp. 947-963, 2001.

[13] S. Gandhani, R. Bhujade and A. Sinhal, "AN IMPROVED AND EFFICIENT IMPLEMENTATION OF CBIR SYSTEM BASED ON COMBINED FEATURES," IET, pp. 353-359.

[14] S. Muhammad Hayat Khan, . D. Fakhri and Dr.Imad Fakhri Taha Alshaikhl, "Comparative study on Content-Based Image Retrieval (CBIR)," IEEE, pp. 61-66, 2013.

[15] Sreedevi S. and Shinto Sebastian, "Fast Image Retrieval with Feature Levels," IEEE, 2013.

[16] S. Ezekiel, Mark G. Alford, David Ferris, Eric Jones,, Adnan Bubalo, Mark Gorniak and Erik Blasch, "Multi-Scale Decomposition Tool for Content Based Image Retrieval," IEEE, 2013.

[17] K. Juneja, A. Verma, S. Goel and S. Goel , "A Survey on Recent Image Indexing and Retrieval Techniques for Lowlevel Feature Extraction in CBIR systems," IEEE, pp. 67-72, 2015.

[18] Khadidja BELATTAR and Sihem MOSTEFAI , "CBIR using Relevance Feedback:Comparative Analysis and Major Challenges," IEEE, pp. 317-325, 2013.

[19] D. Jeyabharathi and D. Suruliandi , "Performance Analysis of Feature Extraction and Classification Techniques in CBIR," IEEE, pp. 1211-, 2013.

[20] Hui Xie, Ying Ji and Yueming Lu, "An Analogy-Relevance Feedback CBIR Method Using Multiple Features," IEEE, pp. 83-86, 2013.

[21] B. Kaur and S. Jindal, "An implementation of Feature Extraction over medical images on OPEN CV Environment".

[22] S. Kumar, S. Jain and T. Zaveri, "PARALLEL APPROACH TO EXPEDITE MORPHOLOGICAL FEATURE EXTRACTION OFREMOTE SENSING IMAGES FOR CBIR SYSTEM," IEEE, pp. 2471-2474, 2014.

[23] Khadidja BELATTAR and Sihem MOSTEFAI , "CBIR with RF:which Technique for which Image," IEEE, 2014.

[24] G. Raghuwanshi and V. Tyagi, "Texture image retrieval using adaptive tetrolet transforms," Elsevier, pp. 1-8, 2015. 